\title{
Effect of transplantation of cardiac stem cells overexpressing integrin-linked kinase on cardiac function of rats with acute myocardial infarction
}

\author{
FENGLI ZHANG* and FENGYAN GUO* \\ Department of Cardiology, Weifang People's Hospital, Weifang, Shandong 261041, P.R. China
}

Received February 1, 2018; Accepted March 30, 2018

DOI: $10.3892 /$ etm.2018.6198

\begin{abstract}
In the present study, we aimed to investigate the effect of transplantation of cardiac stem cells (CSCs) overexpressing integrin-linked kinase (ILK) on cardiac function of rats with acute myocardial infarction (MI). A total of 60 rats were randomly divided into normal saline (NS) group $(\mathrm{n}=20)$, green fluorescent protein (GFP)-CSC group $(n=20)$ and ILK-CSC group ( $\mathrm{n}=20)$. In the ILK-CSC group, CSCs in rats were transfected with GFP adenovirus vector overexpressing ILK. The rat model of MI was established. The cardiac function 4 weeks after transplantation was detected via echocardiography, and the exhaustive swimming experiment was performed to observe the exercise load capacity. Moreover, $\mathrm{Ki}-67$ and $\mathrm{P}-\mathrm{H} 3$ proteins in myocardial tissues of rats were detected via immunohistochemistry, and the expression of GFP was observed under a fluorescence microscope. Cells in the GFP-CSC group were transfected with the empty GFP adenovirus, while those in NS group were not transfected, and other treatments in these two groups were the same as those in the ILK-CSC group. Four weeks after transplantation, left ventricular end-systolic diameter (LVESD) and left ventricular end-diastolic diameter (LVEDD) of rats in the ILK-CSC group were smaller than those in the GFP-CSC group, but left ventricular ejection fraction (LVEF) $(69.88 \pm 5.61 \mathrm{~mm})$ was higher than that in the GFP-CSC group $(\mathrm{P}<0.05)$. The exercise time in the ILK-CSC group (12.69 $\pm 0.58 \mathrm{~min})$ was longer than that in the GFP-CSC and NS groups $(\mathrm{P}<0.05)$. The expression levels of Ki-67 and $\mathrm{P}-\mathrm{H} 3$ proteins in myocardial cells of rats in the ILK-CSC group were higher than those in the GFP-CSC and NS groups $(\mathrm{P}<0.05)$. The number of transplanted cells retained around the infarct region in the ILK-CSC group 3 days after transplantation was obviously larger than that in
\end{abstract}

Correspondence to: Dr Fengyan Guo, Department of Cardiology, Weifang People's Hospital, 151 Guangwen Street, Weifang, Shandong 261041, P.R. China

E-mail: fjr6u2@163.com

${ }^{*}$ Contributed equally

Key words: integrin-linked kinase, cardiac stem cells, acute myocardial infarction, cardiac function the GFP-CSC group $(\mathrm{P}<0.001)$. Intramyocardial injection of CSCs overexpressing ILK immediately after the establishment of rat model of MI can promote myocardial cell proliferation, improve cardiac function and increase exercise capacity of rats.

\section{Introduction}

Myocardial infarction (MI), also known as myocardial infarct, refers to local myocardial necrosis due to ischemia after interruption of blood flow caused by coronary occlusion, and it is a kind of heart disease seriously threatening human healthy, which can lead to such complications as arrhythmia, heart failure or shock $(1,2)$. The traditional treatment methods of MI include drug therapy, interventional therapy and coronary artery bypass grafting. However, these treatment methods bring different degrees of physical or psychological burden to patients due to poor efficacy or complications (3-6). Poulos (7) studied and showed that stem cell transplantation can promote the regeneration of myocardial cells in the local infarct region, thereby repairing the heart and improving the cardiac function. At present, the main stem cells applied include bone marrow mesenchymal, hematopoietic, embryonic and cardiac stem cells (CSCs) (8-11), among which CSC, with strong proliferation and differentiation capacities, is an innate undifferentiated cell derived from the heart (12). CSCs possess the regenerative potential and appear in the myocardial ischemic region in animal model experiment, which can be directionally differentiated into myocardial, vascular smooth muscle and vascular endothelial cells and fibroblasts, thereby participating in repairing the cardiac function $(5,9)$. CSCs are characterized by low tissue specificity and immunogenicity, so their therapeutic effect in transplantation is better than those of other stem cells $(9,10,13)$. Integrin-linked kinase (ILK) is a kind of serine/threonine protein kinase (14), which is highly expressed in the heart and involved in various pathophysiological processes, such as myocardial proliferation, contraction and hypertrophy, and plays an important role in cardiac transplantation and repair (15-17).

In this study, the rat model of MI was established, CSCs in rats were transfected with green fluorescent protein (GFP) adenovirus vector overexpressing ILK, and the effects of transplantation of CSCs overexpressing ILK on cardiac function, exercise capacity and myocardial cell proliferation of rats 
with acute MI were investigated, so as to provide a certain basis for the new therapeutic direction of acute MI.

\section{Materials and methods}

Experimental animals and reagents. Male Sprague-Dawley (SD) rats of clean grade, aged 49-56 days old and weighing 250-300 g, were purchased from the Laboratory Animal Center of Guangzhou University of Traditional Chinese Medicine (Guangzhou, China). ILK complementary deoxyribonucleic acid (cDNA) adenovirus vector was purchased from Invitrogen (Invitrogen; Thermo Fisher Scientific, Inc., Waltham, MA, USA). Lipofectamine 2000, phosphate-buffered saline (PBS), type II collagenase, trypsin, Dulbecco's modified Eagle's medium (DMEM)/Ham's F12 medium, and Iscove's modified Dulbecco's medium (IMDM) were bought from Gibco (Gibco; Thermo Fisher Scientific, Inc.). Rabbit anti-rat ILK, rat Ki-67 and P-H3 antibodies, and horseradish peroxidase-labeled goat anti-rat immunoglobulin $\mathrm{G}(\mathrm{IgG})$ were bought from Abcam (Cambridge, MA, USA).

The study was approved by the Ethics Committee of Weifang People's Hospital (Weifang, China).

Isolation, culture and adenovirus transfection of CSCs. Male SD rats (aged 49-56 days) were anesthetized by an intraperitoneal injection of 3\% sodium pentobarbital (at a dose of $30 \mathrm{mg} / \mathrm{kg}$ ), and sacrificed via cervical dislocation. The rats were housed in pairs in a temperature-controlled room $\left(21-22^{\circ} \mathrm{C}\right)$ with 40-50\% humidity under a 12-h light/dark cycle and were allowed free access to food and water. The large artery was removed from the heart under sterile conditions, and the heart was placed in a petri dish, cut into pieces and rinsed with PBS. Then $0.2 \%$ trypsin and type II collagenase were added at $37^{\circ} \mathrm{C}$ for $5 \mathrm{~min}$, and the operation was repeated 3 times. After trypsin was removed, the pieces were washed twice with complete culture solution (IMDM containing 10\% fetal bovine serum, $100 \mathrm{U} / \mathrm{ml}$ penicillin, $100 \mu \mathrm{g} / \mathrm{ml}$ streptomycin, $2 \mathrm{mmol} / \mathrm{l}$ glutamine and $0.1 \mathrm{mmol} / \mathrm{l} 3$-mercaptoethanol). The above culture solution was added into a culture flask for culture. After 1 week, the pieces were rinsed twice with PBS, and the exfoliated cells were collected and inoculated into the culture flask at a density of $1 \times 10^{5}$ cells $/ \mathrm{ml}$, followed by culture using the mixed culture solution (35\% IMDM, 65\% DMEM and Ham's F-12). When $80 \%$ CSCs were fused, the medium was replaced, and Ad-ILK (GFP adenovirus overexpressing ILK) or Ad-null (empty GFP adenovirus) was added at $37^{\circ} \mathrm{C}$ for $2 \mathrm{~h}$. Finally, the cells were collected after $48 \mathrm{~h}$ of culture.

Construction of rat model of MI and cell transplantation. A total of $60 \mathrm{SD}$ rats were randomly divided into $\mathrm{MI}+$ normal saline group (NS group, $n=20$ ), MI + CSC transfected with empty vector group (GFP-CSC group, $n=20$ ) and $\mathrm{MI}+\mathrm{CSC}$ transfected with ILK group (ILK-CSC group, $n=20$ ). The rat model of MI was constructed via ligation of the left anterior descending coronary artery. At $15 \mathrm{~min}$ after successful modeling, $30 \mu 1 \mathrm{NS}$, GFP-CSC $\left(1 \times 10^{5}\right.$ cells/30 $\left.\mu 1\right)$ and ILK-CSC $\left(1 \times 10^{5}\right.$ cells/30 $\mu$ l) were injected into the MI region at three different points. Then muscles and skin were sutured, and penicillin was intramuscularly injected to prevent infection at 1 week after operation.
Echocardiography method. Four weeks after modeling, all rats underwent echocardiography using the Philips SONOS 5500 color ultrasonic apparatus. After rats were anesthetized via intraperitoneal injection of 3\% sodium pentobarbital $(30 \mathrm{mg} / \mathrm{kg})$, the chest hair was shaved off under a supine position, and then left ventricular end-systolic diameter (LVESD), left ventricular end-diastolic diameter (LVEDD) and left ventricular ejection fraction (LVEF) of rats in each group were measured. The measurement was repeated for 3 times and the average was taken.

Exhaustive swimming experiment. Four weeks after transplantation, exhaustive swimming experiment was performed for all rats to detect the exercise capacity. The rat tails were loaded with the weights weighing $5 \%$ of its weight for weight-bearing swimming in a water tank [45 cm (depth) x $1 \mathrm{~m}$ (length) $\mathrm{x} 1 \mathrm{~m}$ (width)]. The sinking of rats in swimming was the main focus, and the swimming time of rats was recorded.

Observation of the survival of transplanted cells. Three days and 4 weeks after transplantation, 5 rats were sacrificed, respectively, and heart specimens were frozen and stored. The specific method is as follows: the heart tissues were rewarmed to $-20^{\circ} \mathrm{C}$. The left and right ventricles were separated along the ventricular wall, the myocardial tissues in the infarct region and marginal infarct region of 3 rats in each group were collected, fixed in $4 \%$ paraformaldehyde solution, and embedded in paraffin. The paraffin-embedded tissue was sliced into $5 \mu \mathrm{m}$-thick sections. The GFP expression was observed under a fluorescence microscope (BX53; Olympus, Tokyo, Japan) and analyzed using SigmaScan software.

Detection of Ki-67 and P-H3 protein expressions in myocardial tissues of rats via immunohistochemistry. Afterechocardiography and exhaustive swimming experiment, 5 rats were sacrificed in each group, respectively. After heart tissues were prepared into paraffin-embedded sections, they were dewaxed, incubated with $3 \% \mathrm{H}_{2} \mathrm{O}_{2}$ for $10 \mathrm{~min}$, washed with PBS for 3 times (3 min/time). After treatment with $0.5 \%$ Triton X-100 for 30 min, sections were washed with PBS for 3 times ( 3 min/time) and sealed in $10 \%$ goat serum for $30 \mathrm{~min}$. After the serum was removed, rabbit polyclonal Ki-67 antibody (diluted at 1:100; ab833), rabbit polyclonal Histone H3 (phospho S28) antibody (diluted at 1:100; ab5168), and goat anti-rabbit IgG H\&L (Biotin) (ab6720) (dilution: 1:500) (all purchased from Abcam), were added dropwise, and the sections were placed in a wet box at $4^{\circ} \mathrm{C}$ overnight and washed again with PBS for 3 times ( $3 \mathrm{~min} /$ time). After the secondary antibody horseradish peroxidase-labeled goat anti-rat immunoglobulin $\mathrm{G}(\mathrm{IgG})$ was added for incubation at room temperature for $2 \mathrm{~h}$, the sections were washed with PBS for 3 times ( $3 \mathrm{~min} /$ time), followed by color development via diaminobenzidine and hematoxylin counterstaining. Finally, the sections were rinsed with PBS and deionized water and sealed, and the brown-yellow particles with positive protein expression in myocardial cells were observed under the fluorescence microscope (BX53; Olympus). The optical density value was measured using Image-Pro Plus 6.0 software.

Statistical analysis. Statistical analysis was performed using Statistical Product and Service Solutions (SPSS) 13.0 
Table I. Detection results of cardiac function of rats in each group mean \pm standard deviation (SD).

\begin{tabular}{lcccc}
\hline Groups & $\begin{array}{c}\text { Rats } \\
(\mathrm{n})\end{array}$ & $\begin{array}{c}\text { LVESD } \\
(\mathrm{mm})\end{array}$ & $\begin{array}{c}\text { LVEDD } \\
(\mathrm{mm})\end{array}$ & $\begin{array}{c}\text { LVEF } \\
(\%)\end{array}$ \\
\hline NS & 17 & $7.01 \pm 0.48$ & $11.19 \pm 0.52$ & $50.17 \pm 4.08$ \\
GFP-CSC & 14 & $5.85 \pm 0.21^{\mathrm{a}}$ & $8.96 \pm 0.57^{\mathrm{a}}$ & $56.70 \pm 4.25^{\mathrm{a}}$ \\
ILK-CSC & 14 & $4.11 \pm 0.28^{\mathrm{a}, \mathrm{b}}$ & $7.16 \pm 0.44^{\mathrm{a}, \mathrm{b}}$ & $69.88 \pm 5.61^{\mathrm{a}, \mathrm{b}}$ \\
F value & & 6.899 & 12.561 & 18.108 \\
P-value & & 0.012 & $<0.001$ & $<0.001$ \\
\hline
\end{tabular}

${ }^{\text {ap }}<0.05$ vs. NS group; ${ }^{\text {P }}<0.05$ vs. GFP-CSC group. LVESD, left ventricular end-systolic diameter; LVEDD, left ventricular end-diastolic diameter; LVEF, left ventricular ejection fraction; NS, normal saline; GFP, green fluorescent protein; CSC, cardiac stem cell; ILK, integrin-linked kinase.

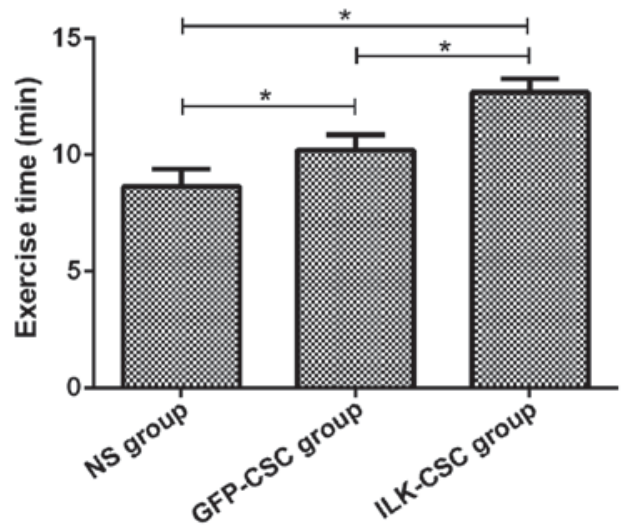

Figure 1. Measurement results of the exercise capacity of rats in each group. Results of the exhaustive swimming experiment show that the exercise time in the ILK-CSC group $(12.69 \pm 0.58 \mathrm{~min})$ is longer than that in the GFP-CSC $(10.21 \pm 0.65 \mathrm{~min})$ and NS $(9.65 \pm 0.73 \mathrm{~min})$ groups, and differences are statistically significant $(* \mathrm{P}<0.05)$. In addition, the exercise time in the GFP-CSC group is longer than that in the NS group ( $\left({ }^{*} \mathrm{P}<0.05\right)$. ILK, integrin-linked kinase; CSC, cardiac stem cell; GFP, green fluorescent protein; NS, normal saline.

software (SPSS, Inc., Chicago, IL, USA). Analysis of variance or t-test was used for quantitative data in line with normal distribution, and Student-Newman-Keuls (SNK)-Q test was used for the pairwise comparison after analysis of variance. ANOVA followed by post hoc test (Dunnett's test) was used to evaluate the quantitative data. All P-values indicated the two-tailed probability, and the inspection level $\alpha=0.05$.

\section{Results}

Four weeks after transplantation of the myocardial cells, 5 rats died of heart failure, including 3 rats in the NS group, 1 rat in the GFP-CSC group and 1 rat in the ILK-CSC group. Finally, 17 rats in the NS group, 14 rats in the GFP-CSC group and 14 rats in the ILK-CSC group were analyzed.

Detection results of cardiac function of rats. Four weeks after transplantation, LVESD and LVEDD of rats in the ILK-CSC group were smaller than those in the GFP-CSC group $(\mathrm{P}<0.05)$, but LVEF was higher than that in the GFP-CSC group $(\mathrm{P}<0.05)$.

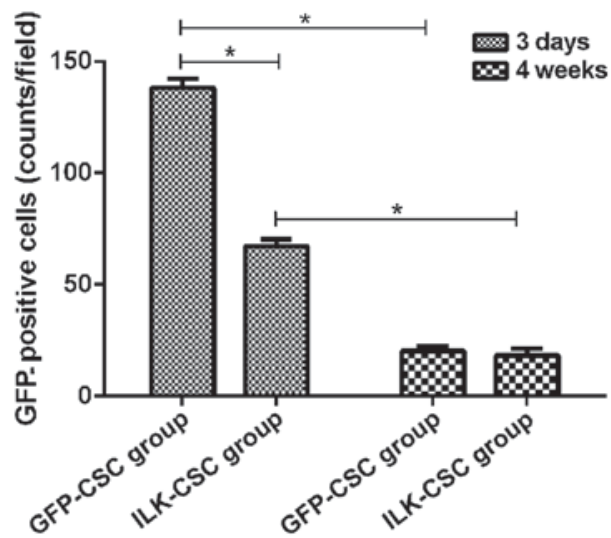

Figure 2. Survival of the transplanted cells in the peripheral infarct region. According to the observation under the fluorescence microscope, the GFP protein is expressed in the peripheral infarct region in both groups at 3 days after transplantation, and the expression of GFP protein in the GFP-CSC group (138 \pm 4 counts/field) is higher than that in the ILK-CSC group $(67 \pm 3$ counts/field), and there is a statistically significant difference $\left({ }^{*} \mathrm{P}<0.05\right)$. The GFP protein expression in the ILK-CSC $(20 \pm 5$ counts/field) and GFP-CSC (18 \pm 4 counts/field) groups 4 weeks after transplantation are lower than those at 3 days after transplantation ( $\mathrm{P}<0.05)$, and there is no statistically significant difference between the two groups $(\mathrm{P}>0.05)$. GFP, green fluorescent protein; ILK, integrin-linked kinase; CSC, cardiac stem cell.

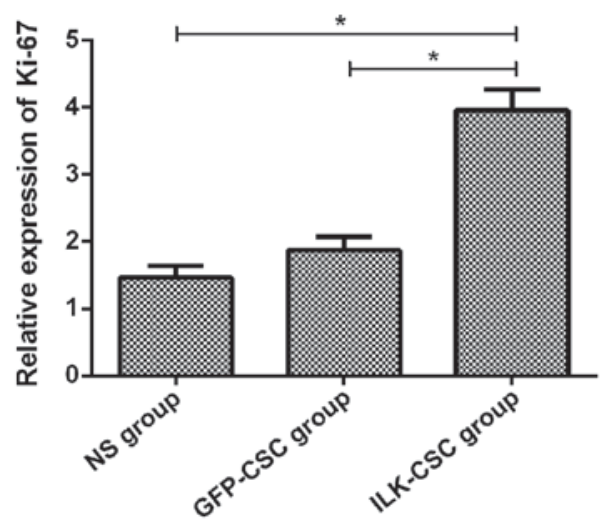

Figure 3. Expression of Ki-67 protein in the myocardial tissues of rats in each group. Results of immunohistochemical staining manifest that the optical density value of Ki-67 protein (3.95 \pm 0.31$)$ in the myocardial tissues of rats in the ILK-CSC group is higher than that in the GFP-CSC $(1.87 \pm 0.20)$ and NS $(1.46 \pm 0.18)$ groups, and differences are statistically significant $\left({ }^{*} \mathrm{P}<0.05\right)$. ILK, integrin-linked kinase; CSC, cardiac stem cell; GFP, green fluorescent protein; NS, normal saline.

In addition, LVESD and LVEDD of rats in the GEP-CSC group were smaller than those in the NS group $(\mathrm{P}<0.05)$, but LVEF was higher than that in the NS group $(\mathrm{P}<0.05)$ (Table I).

Measurement results of exercise capacity of rats in each group. Results of the swimming experiment showed that the exercise time in the ILK-CSC group (12.69 $\pm 0.58 \mathrm{~min})$ was longer than that in the GFP-CSC $(10.21 \pm 0.65 \mathrm{~min})$ and NS $(9.65 \pm 0.73 \mathrm{~min})$ groups, and differences were statistically significant $(\mathrm{P}<0.05)$. In addition, the exercise time in the GFP-CSC group was longer than that in the NS group $(\mathrm{P}<0.05)$ (Fig. 1).

GFP expression in the myocardial tissues in the GFP-CSC and ILK-CSC groups. GFP protein was expressed in the 


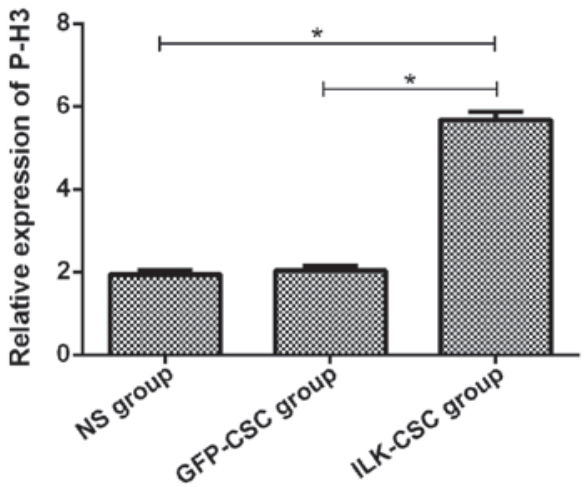

Figure 4. The expression of $\mathrm{P}-\mathrm{H} 3$ protein in the myocardial tissues of rats in each group. Results of immunohistochemical staining manifest that the expression level of $\mathrm{P}-\mathrm{H} 3$ protein $(5.68 \pm 0.19)$ in the myocardial tissues of rats in the ILK-CSC group is higher than that in the GFP-CSC $(2.03 \pm 0.11)$ and NS (1.94 \pm 0.09$)$ groups, and differences are statistically significant $\left({ }^{*} \mathrm{P}<0.05\right)$ ILK, integrin-linked kinase; CSC, cardiac stem cell; GFP, green fluorescent protein; NS, normal saline.

peripheral infarct region in both the GFP-CSC and ILK-CSC groups at 3 days after transplantation, and the expression of the GFP protein in the GFP-CSC group (138 \pm 4 counts/field) was higher than that in the ILK-CSC group (67 \pm 3 counts/field), and there was a statistically significant difference $(\mathrm{P}<0.05)$. The GFP protein expression in the ILK-CSC $(20 \pm 5$ counts/field $)$ and GFP-CSC (18 \pm 4 counts/field) groups 4 weeks after transplantation was lower than those 3 days after transplantation $(\mathrm{P}<0.05)$, and there was no statistically significant difference between the two groups ( $\mathrm{P}>0.05)$ (Fig. 2).

Expression of proliferation-related proteins in myocardial tissues of left ventricle of rats in each group. Immunohistochemical staining was performed for proliferation-related proteins (Ki-67 and $\mathrm{P}-\mathrm{H} 3$ ), and results manifested that the optical density values of $\mathrm{Ki}-67(3.95 \pm 0.31)$ and P-H3 $(5.68 \pm 0.19)$ proteins in the myocardial tissues of rats in the ILK-CSC group were higher than those in the GFP-CSC $(1.87 \pm 0.20,2.03 \pm 0.11)$ and NS $(1.46 \pm 0.18,1.94 \pm 0.09)$ groups, and differences were statistically significant $(\mathrm{P}<0.05)$. The optical density values of the two kinds of proteins had no statistically significant differences between the GFP-CSC and NS groups ( $\mathrm{P}>0.05)$ (Figs. 3 and 4 ).

\section{Discussion}

MI is an issue seriously threatening public health and safety, as well as a global health problem. After MI, irreversible death occurs in myocardial cells, leading to cardiac dysfunction, such as decline in local cardiac contractility, and even heart failure (2). Traditional treatment methods, such as percutaneous transluminal coronary angioplasty, myocardial reperfusion therapy or coronary artery bypass grafting, can improve patients' cardiac function to some extent, but the prognosis is poor (5). CSCs have superior performance of directional differentiation, which can be directionally differentiated into myocardial cells, providing a new choice for cardiac cell transplantation therapy after MI (8). Zhong and Rao (18) studied and showed that after the treatment of ischemic cardiomyopathy with CSC transplantation therapy, the migration and proliferation capabilities of CSCs are superior, there are many new cells and blood vessels in the MI region, and cardiac function recovery is better. ILK can regulate intracellular signaling pathways and transmit extracellular signals into cells, thereby promoting cell survival, reducing apoptosis and promoting cell proliferation and differentiation $(3,19,20)$.

In this study, CSCs in rats were transfected with GFP adenovirus vector overexpressing ILK to investigate the effects of transplantation of CSCs overexpressing ILK on cardiac function, exercise capacity and myocardial cell proliferation of rats with acute MI. During the experimental process, the experimental procedures were strictly implemented, and the scientific, standardized and strict internal quality control was also adopted, so experimental results had high accuracy and reliability. Echocardiography results demonstrated that LVESD and LVEDD of rats in the ILK-CSC group 4 weeks after transplantation were smaller than those in the GFP-CSC group, but LVEF was higher than that in the GFP-CSC group, suggesting that transplantation of CSCs overexpressing ILK can improve cardiac function, and its efficacy is better than that of ordinary CSCs. Moreover, results of the exhaustive swimming experiment revealed that the exercise time in the ILK-CSC group was longer than those in the GFP-CSC and NS groups, indicating that the transplantation of CSCs overexpressing ILK can improve the rats' exercise capacity. Hannigan et al (20) studied and showed that injecting adenovirus overexpressing ILK around the infarct region in rats after acute MI can improve cardiac function, increase myocardial cell proliferation and reduce apoptosis. ILK can protect the myocardial cells of neonatal rats from apoptosis, increase the survival rate of myocardial cells, and improve cardiac function, thereby improving the exercise capacity of rats after transplantation $(21,22)$.

Rats were sacrificed 3 days and 4 weeks after transplantation, and frozen sections were observed under the fluorescence microscope to detect the GFP expression in myocardial tissues in the infarct region. Results demonstrated that the GFP expression was observed in the peripheral infarct region of rats in the IKL-CSC and GFP-CSC groups at 3 days after transplantation, and it was significantly increased in the ILK-CSC group compared with that in the GFP-CSC group, indicating that the high expression of ILK can promote the survival ability of transplanted cells in the peripheral infarct region after MI. Ki-67 is a cell cycle marker that is specifically expressed in proliferating cells (23), and $\mathrm{P}-\mathrm{H} 3$ is a kind of phosphorylated histone that is a marker of cell mitosis, both of which exist in the process of cell DNA synthesis and can reflect the cell proliferation (24). Results of immunohistochemical staining manifested that the expression levels of Ki-67 and P-H3 proteins in the myocardial cells of rats in the ILK-CSC group were higher than those in the GFP-CSC and NS groups, indicating that ILK-CSC transplantation has a stronger effect on activating the proliferation of myocardial cells. In a word, results of this study were basically consistent with those in other studies $(25,26)$. The possible reason for the enhanced proliferation of myocardial cells is that the ILK overexpression contributes to the survival of CSCs in the myocardium, and then CSCs are differentiated into myocardial cells with proliferation capacity, or myocardial cells secrete regulatory factors after the ILK-CSC transplantation, which is beneficial to the survival and proliferation of original myocardial cells (27). 
In conclusion, a rat model of MI was established, and the experiment was carried out on rats transplanted with ILK-CSC in this study. Results indicated that transplantation of CSCs overexpressing ILK can promote proliferation of myocardial cells and restore cardiac function and exercise capacity. Therefore, transplantation of CSCs overexpressing ILK can be used as a new direction in the treatment of MI.

\section{Acknowledgements}

Not applicable.

\section{Funding}

No funding was received.

\section{Availability of data and materials}

The datasets used and/or analyzed during the present study are available from the corresponding author on reasonable request.

\section{Authors' contributions}

FZ drafted this manuscript. FZ and FG contributed to the construction of the rat model of MI and cell transplantation, The exhaustive swimming experiment was performed by both authors. Both authors read and approved the final manuscript.

\section{Ethics approval and consent to participate}

The study was approved by the Ethics Committee of Weifang People's Hospital (Weifang, China).

\section{Consent for publication}

Not applicable.

\section{Competing interests}

The authors declare that they have no competing interests.

\section{References}

1. Desai R,Patel U, Sharma S, Amin P, Bhuva R, Patel MS, Sharma N, Shah M, Patel S, Savani S, et al: Recreational marijuana use and acute myocardial infarction: Insights from nationwide inpatient sample in the United States. Cureus 9: e1816, 2017.

2. Andreadou I, Efentakis P, Balafas E, Togliatto G, Davos CH, Varela A, Dimitriou CA, Nikolaou PE, Maratou E, V, et al: Empagliflozin limits myocardial infarction in vivo and cell death in vitro: Role of STAT3, Mitochondria, and Redox Aspects. Front Physiol 8: 1077, 2017.

3. Dinser L, Meisinger C, Amann U, Heier M, Thilo C, Kuch B, Peters A and Kirchberger I: Peripheral arterial disease is associated with higher mortality in patients with incident acute myocardial infarction. Eur J Intern Med 18: 30007-30004, 2018.

4. Clemens KK, Shariff SZ, McArthur E and Hegele RA: Ezetimibe prescriptions in older Canadian adults after an acute myocardial infarction: A population-based cohort study. Lipids Health Dis 17: 8, 2018.

5. Cimmino G, D'Andrea D, Mauro C, Morisco C and Cirillo P: Treatment of acute myocardial infarction in 2017. G Ital Cardiol (Rome) 18: 3-10, 2017 (In Italian).

6. Noble S and Roffi M: Routine beta-blocker administration following acute myocardial infarction: Why still an unsolved issue? J Thorac Dis 9: 4191-4194, 2017.
7. Poulos J: The limited application of stem cells in medicine: A review. Stem Cell Res Ther 9: 1, 2018.

8. Husby S and Grønbæk K: Mature lymphoid malignancies: Origin, stem cells, and chronicity. Blood Adv 1: 2444-2455, 2017.

9. Li JJ, Liu Y, Zhao J, Li AY and Wang DM: Transplantation of cardiac stem cells overexpressing integrin-linked kinase improves cardiac function in a rat model of acute myocardial infarction. Zhonghua Xin Xue Guan Bing Za Zhi 45: 880-886, 2017 (In Chinese).

10. Sussman MA: Cardiac stem cells: Working independently together. J Am Coll Cardiol 70: 742-744, 2017.

11. Leong YY, Ng WH, Ellison-Hughes GM and Tan JJ: Cardiac stem cells for myocardial regeneration: They are not alone. Front Cardiovasc Med 4: 47, 2017.

12. Lader J, Stachel M and Bu L: Cardiac stem cells for myocardial regeneration: Promising but not ready for prime time. Curr Opin Biotechnol 47: 30-35, 2017.

13. Jackson R, Mount S, Ye B, Mayfield AE, Chan V, Boodhwani M, Davies RA, Haddad H and Davis DR: Isolation of human explant derived cardiac stem cells from cryopreserved heart tissue. PLoS One 12: e0176000, 2017.

14. Zhao J, Liu N, Hao X, Que L, Liu J and Tang X: Association between integrin-linked kinase and hyperthermia in oral squamous cell carcinoma. Oncol Lett 14: 7705-7714, 2017.

15. Olmos G, López-Ongil S and Ruiz Torres MP: Integrin-linked kinase: A new actor in the ageing process? Exp Gerontol 100: 87-90, 2017.

16. Lu W, Xie J, Gu R and Xu B: Expression of integrin-linked kinase improves cardiac function in a swine model of myocardial infarction. Exp Ther Med 13: 1868-1874, 2017.

17. Bai J, Gu R, Wang B, Zhang N, Kang L and Xu B: Overexpression of integrin-linked kinase improves cardiac function in a rat model of doxorubicin-induced chronic heart failure. Zhonghua Xin Xue Guan Bing Za Zhi 42: 225-229, 2014 (In Chinese).

18. Zhong Y and Rao L: Atrioventricular block after reperfusion: A reflection on early beta-blocker therapy for acute myocardial infarction. Anatol J Cardiol 18: 438-439, 2017.

19. Yin Z, Yu B, Liu W and Lan K: Blood transfusion and mortality in myocardial infarction: An updated meta-analysis. Oncotarget 8: 102254-102262, 2017.

20. Hannigan GE, Coles JG and Dedhar S: Integrin-linked kinase at the heart of cardiac contractility, repair, and disease. Circ Res 100: 1408-1414, 2007.

21. Bock-Marquette I, Saxena A, White MD, Dimaio JM and Srivastava D: Thymosin beta4 activates integrin-linked kinase and promotes cardiac cell migration, survival and cardiac repair. Nature 432: 466-472, 2004.

22. Bendig G, Grimmler M, Huttner IG, Wessels G, Dahme T, Just S, Trano N, Katus HA, Fishman MC and Rottbauer W: Integrin-linked kinase, a novel component of the cardiac mechanical stretch sensor, controls contractility in the zebrafish heart. Genes Dev 20: 2361-2372, 2006.

23. Raynov AM, Moon SK, Choung YH, Hong SP and Park K: Nucleoplasm staining patterns and cell cycle-associated expression of $\mathrm{Ki}-67$ in middle ear cholesteatoma. Am J Otolaryngol 26: 296-301, 2005 .

24. López-Sánchez N, Müller U and Frade JM: Lengthening of $\mathrm{G} 2 /$ mitosis in cortical precursors from mice lacking $\beta$-amyloid precursor protein. Neuroscience 130: 51-60, 2005.

25. Mu D, Zhang XL, Xie J, Yuan HH, Wang K, Huang W, Li GN, Lu JR, Mao LJ, Wang L, et al: Intracoronary transplantation of mesenchymal stem cells with overexpressed integrin-linked kinase improves cardiac function in porcine myocardial infarction. Sci Rep 6: 19155, 2016.

26. Mao Q, Lin C, Gao J, Liang X, Gao W, Shen L, Kang L and $\mathrm{Xu} \mathrm{B}$ : Mesenchymal stem cells overexpressing integrin-linked kinase attenuate left ventricular remodeling and improve cardiac function after myocardial infarction. Mol Cell Biochem 397: 203-214, 2014.

27. Ding L, Dong L, Chen X, Zhang L, Xu X, Ferro A and Xu B: Increased expression of integrin-linked kinase attenuates left ventricular remodeling and improves cardiac function after myocardial infarction. Circulation 120: 764-773, 2009.

This work is licensed under a Creative Commons Attribution-NonCommercial-NoDerivatives 4.0 International (CC BY-NC-ND 4.0) License. 